\title{
Near well simulations of heavy oil production with ICD completion
}

\author{
F. S. Moghaddam, R. Aryal, H. Aakre \& B. M. Halvorsen \\ Telemark University College, Norway
}

\begin{abstract}
Heavy oil reservoirs have been proven to be remarkable energy resources due to the high demand in today's oil market. Therefore, increased oil recovery is an essential requirement in fulfilling global energy demand. Oil viscosity is a key factor in determining the rate of production from heavy oil reservoirs. Long horizontal wells are used to obtain maximum reservoir contact. Due to frictional pressure drop along the long well, the driving forces are different from one location to another in the well - this is called the heel-toe effect. In a homogeneous reservoir the oil production rate will be significantly higher in the heel than in the toe, and this will lead to early water or gas breakthrough in the heel. In heterogeneous reservoirs, early breakthrough will occur in the high permeability zones due to low flow resistance in the reservoir. Horizontal wells with passive Inflow Control Devices (ICDs) have shown improved performance in terms of oil production from lateral thin reservoirs. Different types of ICDs are developed to delay the early breakthrough by restricting the flow. The focus area of this paper is the production of heavy oil from a reservoir with water drive and standard nozzle ICD completion. Heavy oil production from a homogeneous reservoir with different viscosities is simulated. Breakthrough time and cumulative oil production is compared. In addition, the effect of ICDs in a heterogeneous reservoir is studied. The results show that standard nozzle ICDs delay the water breakthrough, but are not able to choke the water after breakthrough. The near well simulations are performed with the reservoir model Rocx in combination with OLGA. Tecplot is used to show the flow behaviour in the reservoir.

Keywords: increased oil recovery, ICD, heavy oil, homogeneous/heterogeneous reservoir.
\end{abstract}




\section{Introduction}

Heavy oil reservoirs have been proven to be remarkable energy resources due to the high demand in today's oil market. Heavy oil reservoirs have less value due to their higher viscosity compared to conventional oil resources. The economical situation all around the world and the high availability of heavy oil recourses are the two main reasons which make these resources more valuable than ever before [1]. Horizontal drilling have been developed and utilized successfully during the past decades which enables more efficient production from long thin oil deposits by enhancing reservoir contact and thereby optimizing well performance [2-5]. However, there are still challenges regarding early water or gas breakthrough due to the frictional pressure drop along the well, reservoir heterogeneity and annular flow which impacts the production. The main consequence of frictional pressure drop along the long well is higher production rate in the heel compared to the toe section of the well due to the heel - toe effect (i.e. different driving force from one to another location of the well in horizontal direction). In addition to heel-toe effect, reservoir heterogeneity and annular flow are also limiting the oil recovery [2-5]. Inflow Control Devices (ICDs) have been developed and introduced by Norsk Hydro since early 1990s [2]. ICDs are passive Inflow Control Devices with the main function of equalizing the flow from heel to toe part of the well. With the application of ICDs water and gas breakthrough are delayed for a longer duration and annular flow is decreased [2-5]. However, due to ICDs prefixed installation and dynamic conditions of the reservoir, pressure profile through the well changes with time and therefore leading to early water or gas breakthrough. Further developments in inflow control technology have proven to be successful in solving this problem [5]. The objective of this paper is to evaluate the influence of viscosity and heterogeneity over the well performance with standard nozzle type ICD. In order to achieve this target various simulations have been done by coupling OLGA and Rocx simulating software. Tecplot is used to show the flow behaviour in the reservoir.

\section{Theory}

\subsection{Darcy's law}

Darcy's law described in eqn (1) is the fundamental principal for fluid behaviour through a porous media which describes the physical dependency of different parameters involved in fluid flow [6].

$$
v=-k \frac{1}{\mu} \frac{d P}{d x}
$$

$v$ is the fluid velocity, $k$ is the permeability (Da), $\mu$ is the dynamic viscosity and $\left(\frac{d P}{d x}\right)$ is the pressure gradient. The main assumptions for Darcy's Law include laminar flow and incompressible fluid. 


\subsection{Viscosity}

Viscosity is the most influential fluid property in determining oil recovery and production rate. Heavy oil and extra heavy oil viscosity varies within the range of $10 \mathrm{cP}$ to more than $1000,000 \mathrm{cP}$. In general, the production costs increases with increasing oil viscosity. The hydrocarbon with highest viscosity is known as bitumen and it is in solid phase at room temperature. With increase of temperature it softens gradually. Viscosity of heavy oil, extra heavy oil and bitumen decreases with increase of temperature, as can be seen from Figure 1 [1].

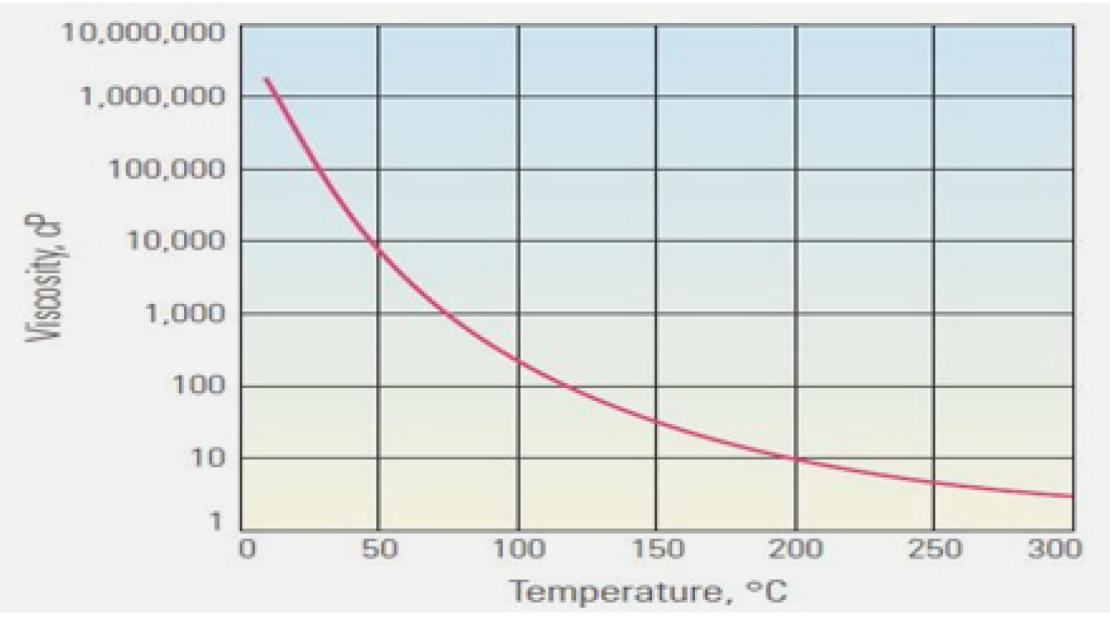

Figure 1: General trend for dependency of heavy oil viscosity with temperature difference [1].

\subsection{Heterogeneity}

A good understanding about reservoir heterogeneity is essential in terms of oil production and recovery. Reservoirs are formed in various environments which results in different characteristics due to the depositional location and conditions. Changes in reservoir properties due to location differences are known as reservoir heterogeneity [6]. In this study, changes in reservoirs permeability are the matter of concern in terms of reservoir heterogeneity. Permeability difference is more likely to appear in long reservoirs, therefore, leading to non-uniform pressure distribution through the well and early water or gas breakthrough [3].

\subsection{Heel-toe effect}

A major challenge in long horizontal wells is heel-toe effect due to the frictional pressure losses along the well. After the production starts, the draw down (i.e. pressure difference between the well bore and reservoir) starts to change differently with higher values in heel compared to toe part of the well shown in 
Figure 2. The considerable changes in the drawdown along the well are leading to early water or gas breakthrough and are reducing the well performance in terms of oil production and recovery [7].

$\Delta P_{h}$ is the drawdown in the heel and $\Delta P_{t}$ is the draw down in the toe part of the well.

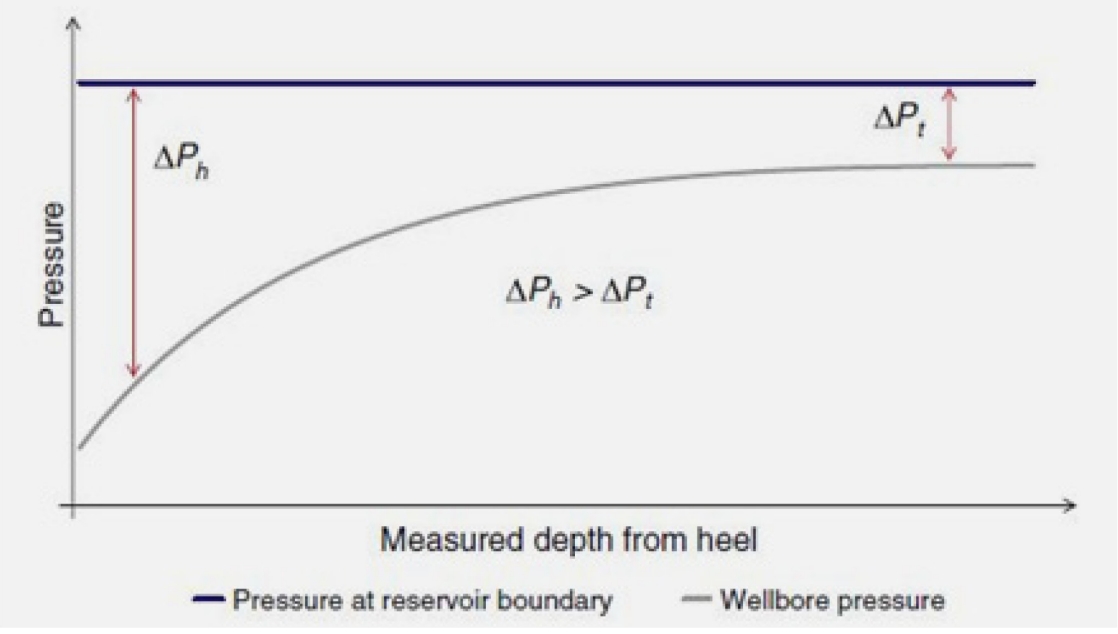

Figure 2: $\quad$ Increase in frictional pressure losses due to the heel-toe effect [7].

\subsection{Development of inflow control technology}

Inflow control technology has been developed during the recent decades in order to increase the oil production and recovery by optimizing the well performance. Early water or gas breakthrough is the major consequence of non-uniform flow rate from different parts of the wells due to the frictional pressure drop and reservoir heterogeneity. Passive Inflow Control Devices (ICDs) are installed on the base pipe in order to equalize the flow by compensating the heel-toe effect and heterogeneity effect. The main downside of passive ICDs is due to their prefixed installation. Passive ICDs cannot adjust themselves with the dynamic reservoir conditions during the oil production. When breakthrough occurs, ICDs do not have the ability to choke or stop the flow of low viscous fluids into the pipeline [2-5]. In order to overcome these downsides for passive ICDs and increase the production rate, further developments such as Autonomous Inflow Control Device (AICD) and Autonomous Inflow Control Valve (AICV) have been introduced to oil industry during the recent years $[5,8]$.

\subsection{Zonal isolation}

Having better control over the production zones by reducing annular flow increases the production and recovery rate to a considerable level. By this mean, zonal isolation is piloted in the annulus with AICD (i.e. one of the successful developments in inflow control technology) shown in Figure 3 [5]. 


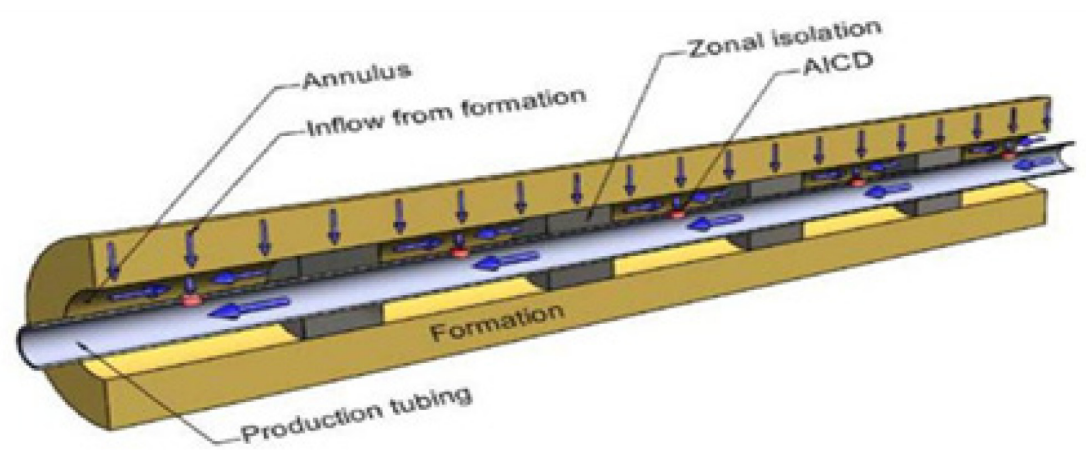

Figure 3: Zonal isolation installed in the annulus with AICD in order to prevent the annular flow [5].

\section{Results}

In this case study the well performance with ICD completion in homogeneous heavy oil reservoir is evaluated. Furthermore we study well performance with ICD completion in heterogeneous heavy oil reservoirs.

\subsection{Near well simulation in homogeneous reservoirs}

In order to evaluate well performance with ICD completion, heavy oil production with 100cP viscosity from a limited section of a homogeneous reservoir was simulated by coupling OLGA and Rocx software. Geometry of the studied section is $49.6 \mathrm{~m}$ in $\mathrm{x}, 80 \mathrm{~m}$ in $\mathrm{y}$ and $20 \mathrm{~m}$ in $\mathrm{z}$ direction. The $2 \mathrm{D}$ schematic view of the reservoir and well with four installed ICDs is present in Figure 4. The distance between the ICDs is $12.4 \mathrm{~m}$. As can be seen from the figure, a pressure difference of 5 bar between the base pipe pressure outlet and the water drive pressure was considered as the driving force for oil production.

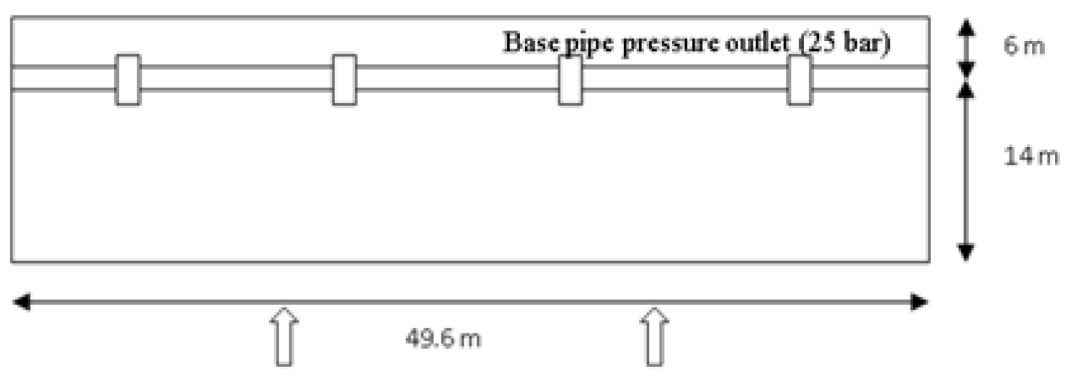

Water drive pressure inlet ( 30 bar)

Figure 4: Schematic view of the reservoir and well with ICD completion in $2 \mathrm{D}$. 
Complete view of the reservoir with grids is shown in Figure 5. The grid spacing in $\mathrm{x}$ and $\mathrm{z}$ directions are constant and in $\mathrm{y}$ direction the grid spacing becomes finer toward the centre.

Phase profiles, showing the reservoir behaviour during production life time of the well until water breakthrough are illustrated in Figures 6 and 7. The figures show the water coning towards the well during the oil production. Water breakthrough occurred after about 6 days of production.

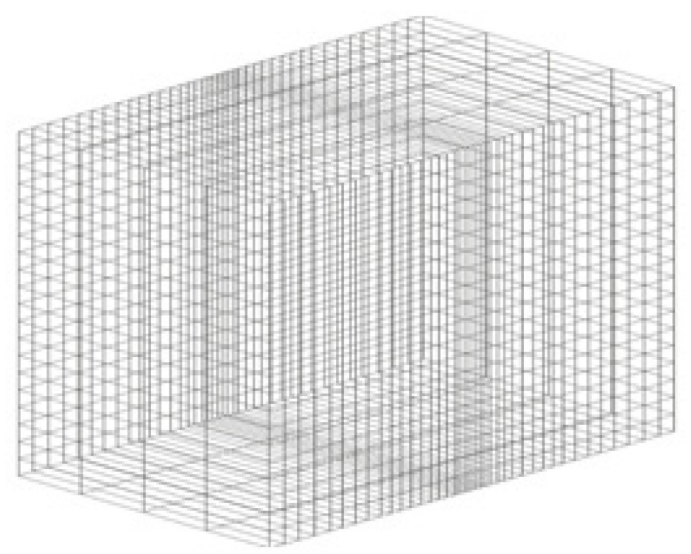

Figure 5: $\quad$ Schematic view of the reservoir with grids in 3D.
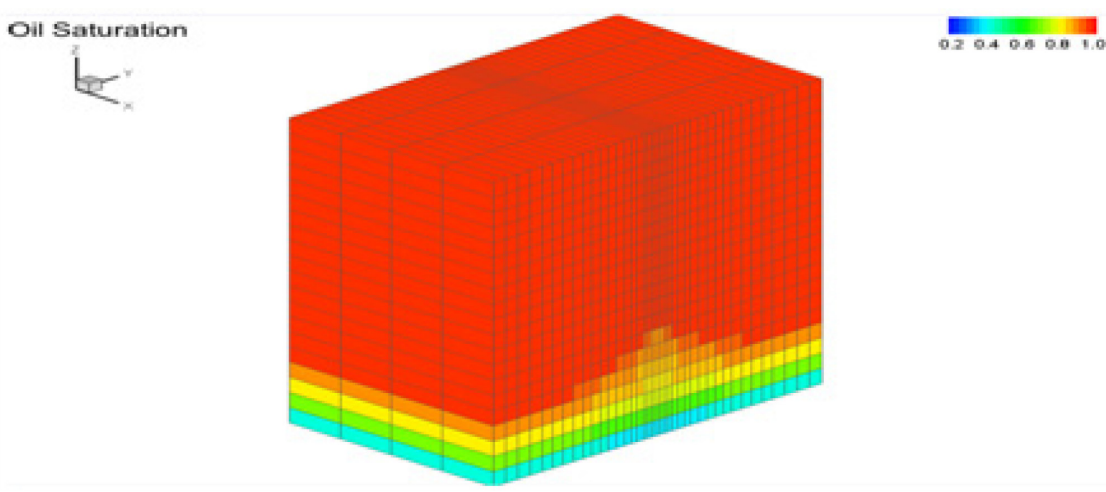

Figure 6: Phase profile at day 3 of the production. 

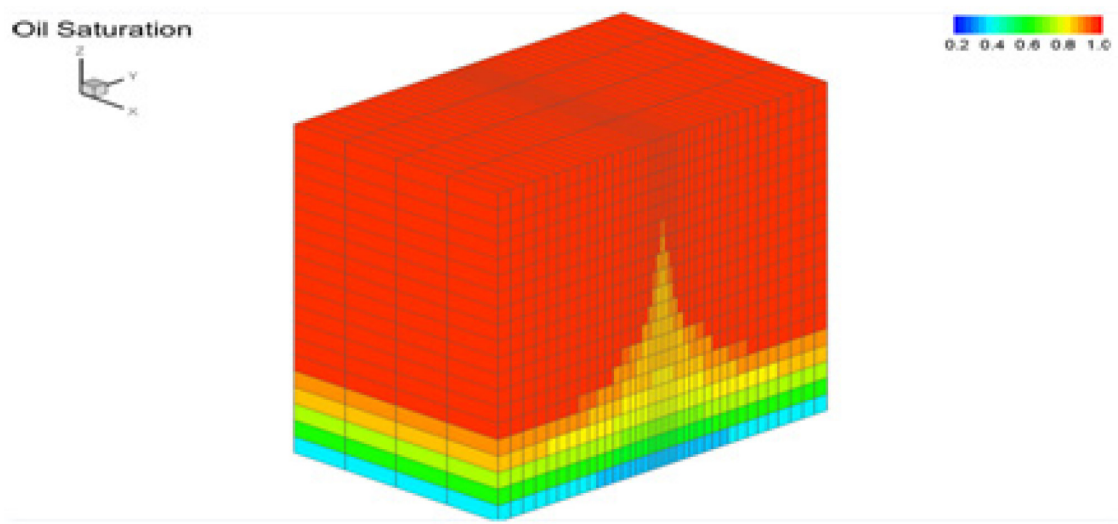

Figure 7: $\quad$ Phase profile at day 6 of the production when water breakthrough occurs.

The breakthrough time can also be observed from the accumulated oil and liquid curves shown in Figure 8. After the water breakthrough occurred at day 6 the total accumulated liquid flow increases significantly due to the high flow rates of water. Oil production is reduced after water breakthrough and after 20 days of production the well is producing about two times more water than oil.

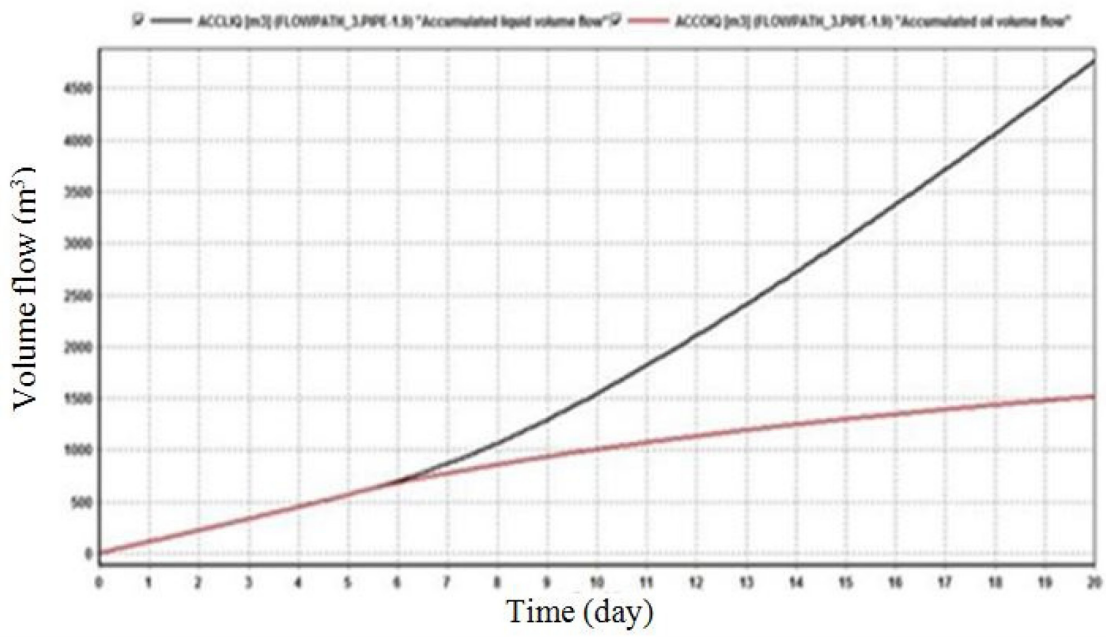

Figure 8: $\quad$ Accumulated curves for liquid volume flow and oil volume flow.

The pressure profile inside the reservoir during the production is presented in Figure 9. The pressure in the reservoir far from the well is about 30bar and decreases gradually to 25bar in the base pipe. 

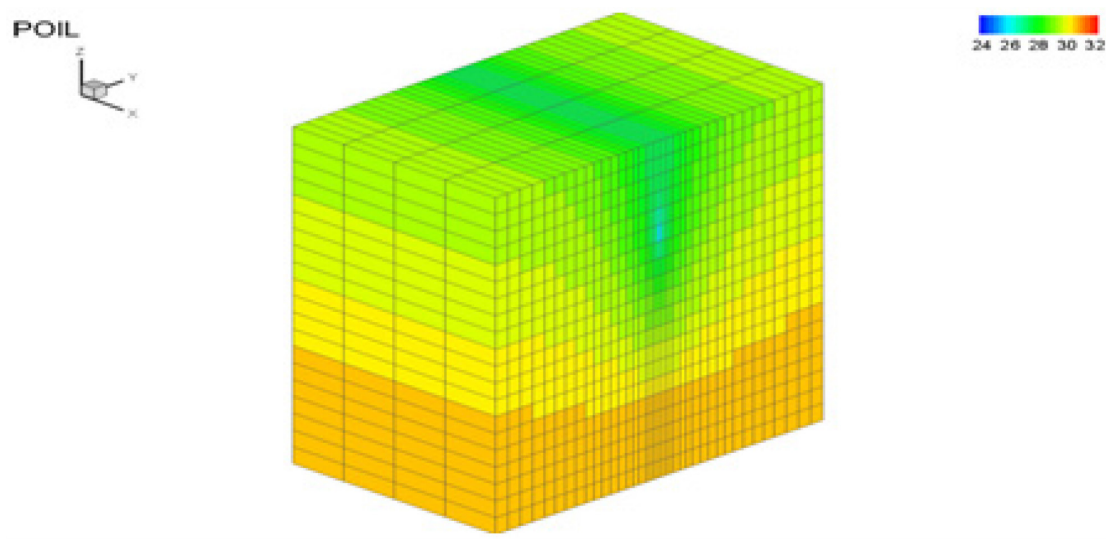

Figure 9: $\quad$ Pressure profile in 3D at second day of the production.

Since only a limited section of the well was studied, the frictional pressure drop cannot be observed. Therefore, pressure profile along the well during the production and before the breakthrough occurred was constant (as shown in Figure 10).

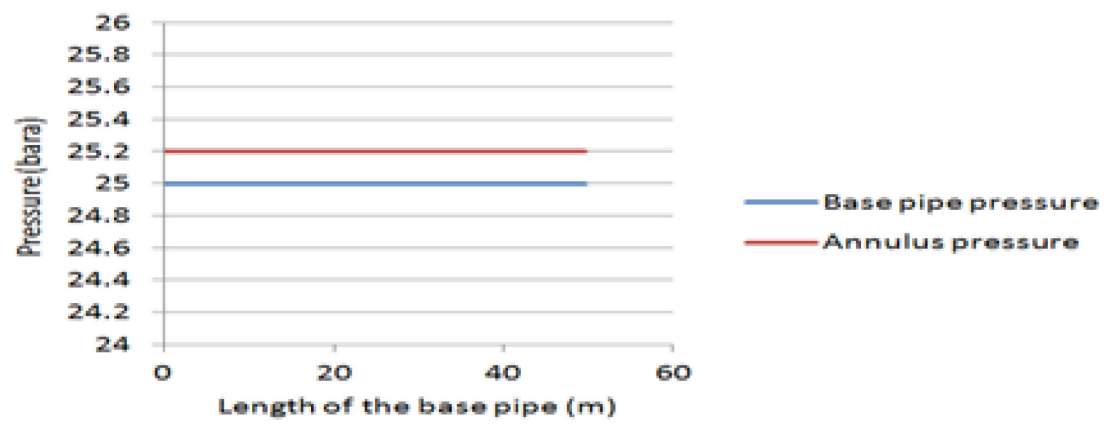

Figure 10: Pressure drop from the annulus to the base pipe, during the production before the breakthrough occurred.

Furthermore, heavy oil with viscosity 500cP was simulated in a reservoir with the same permeability and porosity as the simulated case with $100 \mathrm{cP}$ oil. The water breakthrough occurred after approximately 21 days. The two cases are compared in Figure 11. The accumulated oil volume at the breakthrough time was about $500 \mathrm{~m}^{3}$ for the $500 \mathrm{cP}$ oil and $700 \mathrm{~m}^{3}$ for the $100 \mathrm{cP}$ oil. After breakthrough, the total liquid production increases significantly for both cases due to the high flow rate of water into the well. 


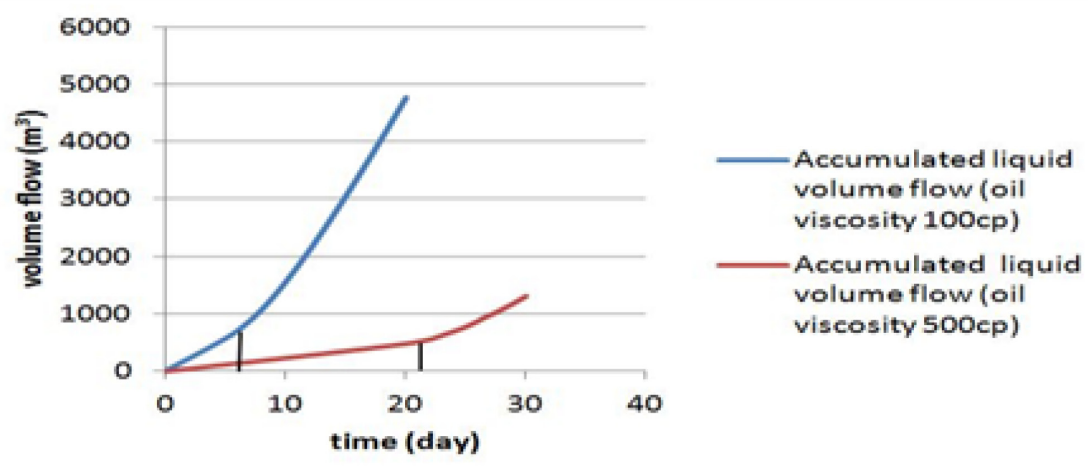

Figure 11: Accumulated liquid volume flow for two cases of oil viscosity with 100cP and 500cP.

\subsection{Near well simulation from heterogeneous reservoirs}

The effect of reservoir heterogeneity on well performance with ICD completion was studied. Heavy oil production with 100cP viscosity from heterogeneous reservoir was simulated and the results were compared to that of the homogeneous case with 100cP oil viscosity. The studied section of the reservoir has the same geometry as the reservoir in previous cases and it was divided into four main sections. The permeability in three sections was 5Da and in one section 50Da. The breakthrough time from heterogeneous reservoir occurred at the second day of the production, which was considerably earlier than the breakthrough time from homogeneous reservoir (Figure 12). As can be observed from the figure the accumulated volume flow at breakthrough time is about $400 \mathrm{~m}^{3}$ which is considerably lower than that of the homogeneous case with approximately $700 \mathrm{~m}^{3}$.

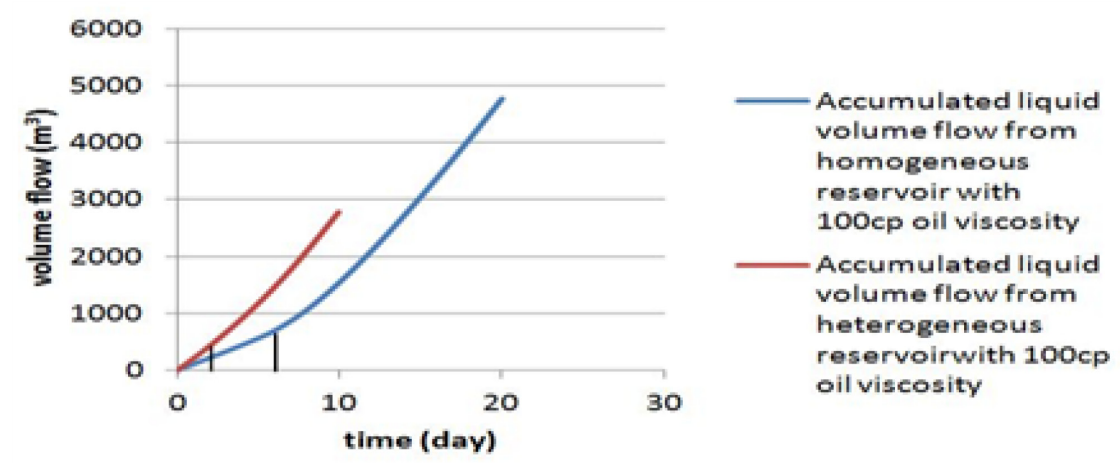

Figure 12: Accumulated liquid volume flow from heterogeneous and homogeneous reservoirs with 100cP oil viscosity. 
Figure 13 illustrates how fast the water permeated through the high permeable region of heterogeneous reservoir and reached the well compared to regions with lower permeability.
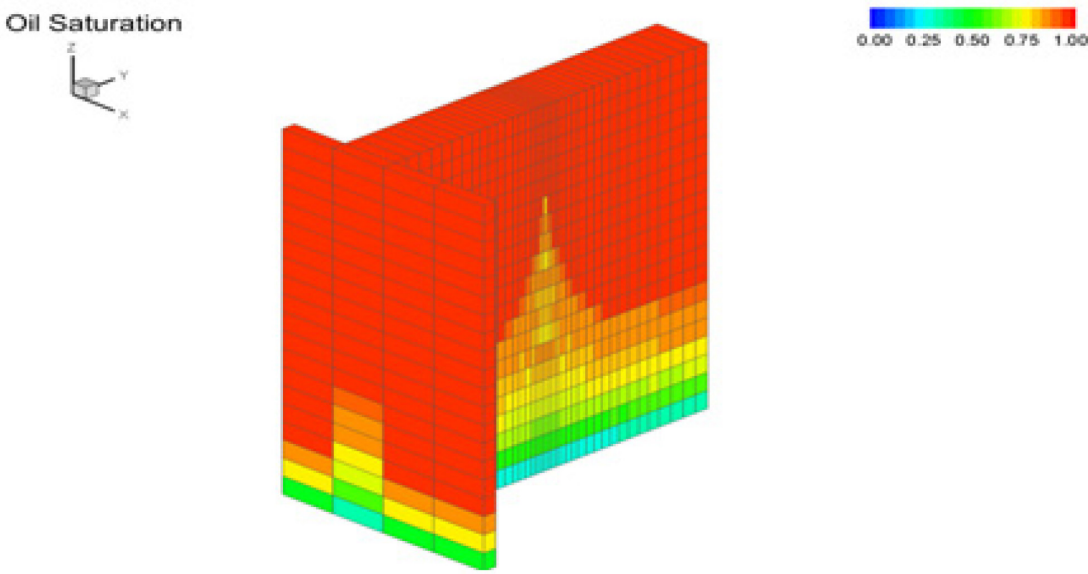

Figure 13: Phase profile comparing heavy oil permeation in high permeable region towards the well with lower permeable region.

\section{Conclusion}

Horizontal wells with passive Inflow Control Devices (ICDs) have increased the oil recovery from reservoirs with thin oil columns. This paper focuses on simulations of heavy oil production from reservoir with water drive and standard nozzle ICD completion. The near well simulations are performed with the reservoir model Rocx in combination with OLGA. Tecplot is used to show the flow behaviour in the reservoir.

Heavy oil production from homogeneous reservoir with oil viscosity of $100 \mathrm{cP}$ and $500 \mathrm{cP}$ is simulated. The permeability was 5 Da. Breakthrough time and cumulative oil production is compared. The water breakthrough occurred after approximately 6 days for the 100cP oil and 21 days for the 500cP oil. The accumulated oil volume at the breakthrough time was about $700 \mathrm{~m}^{3}$ for the $100 \mathrm{cP}$ oil and $500 \mathrm{~m}^{3}$ for the $500 \mathrm{cP}$ oil. After breakthrough the total liquid production increases significantly for both cases due to the high flow rate of water into the well.

In addition the effect of ICDs in a heterogeneous reservoir with $100 \mathrm{cP}$ oil is studied. The permeability in three sections was 5Da and in one section 50Da. The breakthrough time from heterogeneous reservoir occurred at the second day of the production, which was considerably earlier than the breakthrough time from homogeneous reservoir. The accumulated volume flow at breakthrough time was about $400 \mathrm{~m}^{3}$ which is considerably lower than that of the homogeneous case. Standard nozzle ICDs are capable of delaying water breakthrough but are not able to choke the water after breakthrough. 


\section{Nomenclature}

$\begin{array}{ll}\text { ICD } & \text { Inflow Control Device } \\ \text { AICD } & \text { Autonomous Inflow Control Device } \\ \text { AICV } & \text { Autonomous Inflow Control Valve } \\ v & \text { fluid velocity } \\ k & \text { permeability } \\ \mu & \text { dynamic viscosity } \\ \frac{d P}{d x} & \text { pressure gradient due to the change in distance } \\ \Delta P_{h} & \text { drawdown in the heel section of the well } \\ \Delta P_{t} & \text { drawdown in the toe section of the well }\end{array}$

\section{Acknowledgements}

The authors wish to thank Telemark University College for its technical and financial support. We also wish to thank Dr. Vidar Mathiesen and Mahmood Mustafa Alhelol for all their technical support.

\section{References}

[1] Alboudwarej, H., et al. Highlighting heavy oil. Oilfield review, 2006, 18(2): p. 34-53.

[2] Al-Khelaiwi, F. and D. Davies. Inflow control devices: application and value quantification of a developing technology. in International Oil Conference and Exhibition in Mexico. 2007.

[3] Fernandes, P., Z. Li, and D. Zhu. Understanding the Roles of Inflow-Control Devices in Optimizing Horizontal-Well Performance. in SPE Annual Technical Conference and Exhibition. 2009.

[4] Krinis, D., et al. SS-Optimizing Horizontal Well Performance In NonUniform Pressure Environments Using Passive Inflow Control Devices. in Offshore Technology Conference. 2009.

[5] Mathiesen, V., et al., Autonomous Valve, A Game Changer Of Inflow Control In Horizontal Wells. Offshore Europe, 2011.

[6] Ahmed, T., Reservoir engineering handbook. 2006, Amsterdam: Elsevier. $\mathrm{XV}, 1360$ s. : ill.

[7] Birchenko, V.M., K.M. Muradov, and D.R. Davies, Reduction of the horizontal well's heel-toe effect with inflow control devices. Journal of Petroleum Science and Engineering, 2010, 75(1-2): p. 244-250.

[8] Inflow Control AS. Getting more out of oil. Available from: http://www.inflowcontrol.no/. 